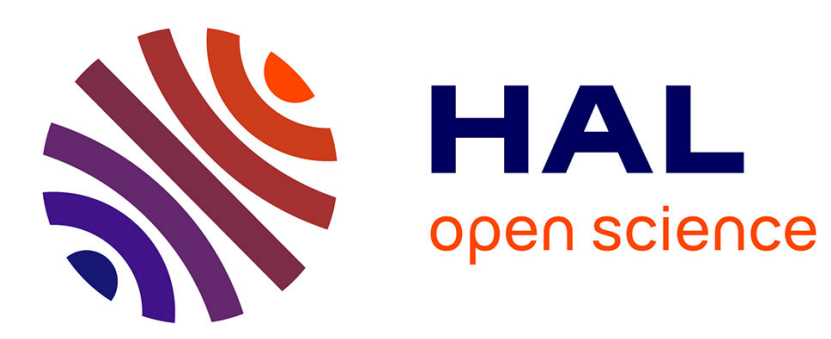

\title{
A 3-variable PDE model for predicting fungal growth derived from microscopic mechanisms
}

Huan Du, Thi-Bich-Thuy Tran, Patrick Perre

\section{To cite this version:}

Huan Du, Thi-Bich-Thuy Tran, Patrick Perre. A 3-variable PDE model for predicting fungal growth derived from microscopic mechanisms. Journal of Theoretical Biology, 2019, 470, pp.90-100. 10.1016/j.jtbi.2019.03.015 . hal-02294135

\section{HAL Id: hal-02294135 \\ https://hal.science/hal-02294135}

Submitted on 22 Oct 2021

HAL is a multi-disciplinary open access archive for the deposit and dissemination of scientific research documents, whether they are published or not. The documents may come from teaching and research institutions in France or abroad, or from public or private research centers.
L'archive ouverte pluridisciplinaire HAL, est destinée au dépôt et à la diffusion de documents scientifiques de niveau recherche, publiés ou non, émanant des établissements d'enseignement et de recherche français ou étrangers, des laboratoires publics ou privés.

\section{(ㄷ)(1) $\$$}

Distributed under a Creative Commons Attribution - NonCommercial| 4.0 International 


\title{
A 3-variable PDE model for predicting fungal growth derived from microscopic mechanisms
}

\author{
Huan Du ${ }^{\mathrm{a}}$, Thi-Bich-Thuy Tran ${ }^{\mathrm{a}}$, Patrick Perréa,b \\ ${ }^{a}$ LGPM, CentraleSupélec, SFR Condorcet FR CNRS 3417, Université Paris-Saclay, Centre Européen de \\ Biotechnologie et de Bioéconomie (CEBB), 3 Rue des Rouges Terres, 51110, Pomacle, France \\ ${ }^{b}$ LGPM, CentraleSupélec, Université Paris-Saclay, 8-10 Rue Joliot-Curie, 91190, Gif-sur-Yvette, France
}

\begin{abstract}
In this work, we present a new PDE model of the growth of Postia placenta, a species of brown rot fungus. The formulation was derived mainly from the biological mechanisms embedded in our discrete model, validated against experimental data. In order to mimic the growth mechanisms, we propose a new reaction-diffusion formulation, based on three variables: the concentration of tips, the branch density and the total hyphal density. The evolution of tips obeys a reaction-diffusion model, with constant diffusivity, while the evolution of the two other variables results from time integrals. The numerical solution is in excellent agreement with the averaged radial tip/hyphal densities of the mycelial network obtained by the discrete model. Thanks to the efficient exponential Euler method with Krylov subspace approximation, the solution needs only $3.5 s$ of CPU time to simulate 104-day of mycelium growth, in comparison with 8 hours for the discrete model. The great reduction of the RAM memory and computing time gives the possibility to upscale the simulation. The novelty of the PDE system is that the spatial colonization is formulated as a diffusion mechanism, which is self-standing, contrary to models based on an advection term. The continuous model can also reproduce the radial densities when the growth parameters in the discrete model are varied to adapt to different growth conditions. The correlation constructed between the two models provides us a tool for mutual insights between local biological mechanisms to the global biomass distribution, especially when analyzing experimental data.
\end{abstract}

Keywords: Reaction-diffusion, continuum modeling, upscaling, fungus

\section{Introduction}

Wood and wood-based products have been popular constructive materials due to their excellent structural properties for many years. The good thermal performance and design of new manufacturing technology enable the reduction of energy consumption and emission, which addresses the

\footnotetext{
*Patrick Perré

Email address: patrick.perre@centralesupelec.fr (Patrick Perré)
} 
5 present needs in building construction. This is why these materials became even more promoted in construction. However, fungal decay in bio-based materials is of major concern in relation to the service time of buildings and even to human safety, since it weakens the structural support of wood enough to cause mechanical failure. Every year an enormous amount of wood and woodbased products is destroyed by fungal decay, among which brown rot fungi are the most common and destructive within buildings throughout Europe and North America due to their rapid decay mechanisms [1]. Many researches have studied the growth and decay patterns of brown rot fungi as well as the impact of environmental factors to their growth $[2,3,4,5,6,7,8]$. Nevertheless, the laboratory observations are constrained by the scales of study and their cost. As a supplementary tool, mathematical modeling in combination with laboratory experiments can realize a deeper and wider insight in a more efficient way.

The modeling techniques to simulate the spatial distribution of fungi are classified into two categories: discrete models and continuous models. The previous ones adapt well to model the mycelial growth at a small scale in identifying individual hypha to form mycelial networks in different environments. For example, Boswell et al. (2007) and Boswell (2008) respectively developed a two-dimensional (2D) and a three-dimensional (3D) lattice-based model to simulate mycelial growth in a soil-like environment [9, 10]. Fuhr et al. (2011) constructed a 3D lattice-free model of hyphal growth in the heartwood of Norway spruce in simplifying the structure of wood [11]. These models are generally constrained in a small scale by the necessary RAM memory and the computing time. In contrast, the continuous models provide a potential of modeling the fungal growth from the colony-scale to the macroscale, in which the density of fungal matter was viewed as averaged quantities. The early models were extended from reaction-advection systems which included nutrients and geometries of mycelial spread $[12,13,14,15]$. However, the advection process requires the development direction to be known at each point, which limits the prediction ability of the code and/or augments the mathematical complexity especially in heterogeneous media. Thus, in the recent models, the diffusion process has replaced the advection to model the movement of hyphal biomass $[16,17,18,19,20,21]$ or combined with the advection process to model the movement of tips and hyphae [22, 23]. However, in these models, some of the mechanisms were based on assumptions and no precise validation process has been carried out.

The strategy of our work is to enlarge the spatio-temporal scale of the study of fungal growth step by step in an accurate and efficient way. Thus, we started from the micro-scale experiment [24] to observe the hyphal growth behaviors of a species of the brown rot fungi, Postia placenta. Then, a discrete model [25], derived from the biological mechanisms of hyphal development, was precisely 

mycelial growth at a larger spatial-scale in much shorter time. However, to reach the macroscale with an even a higher computational efficiency, a continuous model is needed.

In this work, we propose a PDE formulation mainly derived from the mechanisms of hyphal growth at the microscopic level. A reaction-diffusion equation mimics the movement and proliferation of tips. Then, the evolution of hyphal biomass is related to the tip density following the tip elongation mechanism. Most of parameters of the continuous model were directly obtained from the discrete model, while three remaining parameters were determined by an inverse procedure: the model parameters were adjusted to minimize the objective function, i.e. the gap between the PDE profiles and the radial profiles of hyphal density calculated by the validated discrete model. Thanks to the exponential Euler method with Krylov subspace approximation, the model was accurately solved in an efficient way. Then, the growth parameters in the discrete model have been varied to investigate their influences to the distributions of tips and hyphae. The good agreements between the numerical solutions and the profiles obtained in the discrete model verified the excellent prediction capacity of the continuous model and its facility to adapt to different growth conditions. Additionally, thanks to the good correspondence of the two models, the local mechanisms of the mycelial network can also be estimated via the change of biomass distribution. Furthermore, due to the pure diffusion process, this model is simple enough to extend to study the fungal growth in multi-dimensional spaces and in porous media, and even provides a base to the multi-scale modeling of the fungal development in wood.

\section{Method and materials}

\subsection{Discrete mechanisms and radial profiles}

In our discrete model [25], three main microscopic mechanisms (i.e., tip elongation, branching and anastomosis) of hyphal growth were implemented in a regular square-lattice to simulate the development of a mycelial network. The two fundamental features of mycelial growth are tips and hyphae. Tips belong to two classes: active tips which extend at a certain, non-null, rate and dormant tips which do not elongate. A hypha elongates by the extension of an active tip, following its dominant direction. The elongation rate of each active tip follows a corrected gamma distribution. Each site of the lattice can contain numerous hyphae and/or tips. Lateral and apical branching are considered as way to produce new tips. The rate of lateral branching depends on the lateral branching probability and the total number of tips and the lateral branches are uniformly distributed throughout the mycelium. The apical branching occurs to the active tips and its rate 
is determine by the apical branching probability. The branching is inhibited in three special cases:

75 (i) no lateral branches emerge in the proximity of an existed branch; (ii) the formation of a lateral branch around a hyphal tip is inhibited by apical dominance; (iii) the apical branching does not occur if its trailing hypha has not a certain length. Apart from the process of tip proliferation, tips can also disappear through anastomosis. The fusion of a tip with a hypha can occur when they contact each other to yield an interconnected mycelial network.

This model has been validated by a precise process using the experimental data of the growth of P. Placenta during 17 days. With the determined parameters, the spatio-temporal development of a mycelial network was generated from a spore during a longer timescale of $9 \times 10^{6} s(\sim 104$ days). We divided the mycelial network into a series of rings with a gap of $\Delta r=1.5 \times 10^{-4} \mathrm{~m}$, of which the radial densities of tips and hyphae were respectively calculated by averaging the discrete network over radius increments. The tip density is defined as the number of tips per unit volume, while the hyphal density denotes the length of hyphae per unit volume. Due to the numerous stochastic processes in the discrete model, a single realization contains fluctuations. Therefore, we repeated the simulations 20 times with the same parameters and averaged the two resulting densities for each ring: (i) the averaged tip density denoted by $\rho_{t i p}(r, t)$ in the ring around the radius $r$ and at time $t$ and (ii) the averaged hyphal density denoted by $\rho_{\text {hypha }}(r, t)$. In the following, the temporal evolution of the tip and hyphal distributions, the two averaged densities, were calculated at nine instants from $t=2.25 \times 10^{5}$ to $t=9 \times 10^{6} \mathrm{~s}$.

\subsection{Continuous model}

A continuous model was directly derived from the three microscopic mechanisms of mycelial growth of the above-presented discrete model. The fungal mycelium is represented by two components: tip density and hyphal density, which represent respectively the proliferation capacity of the mycelium and the total biomass quantity. The tip density, denoted by $C(\mathbf{x}, t)$, is the number of tips in a unit volume at the spatial position $\mathbf{x}$ and time $t$, while $B(\mathbf{x}, t)$ is the hyphal density defined as the length of hyphae per unit volume.

The three main mechanisms of mycelial growth, tip elongation, branching and anastomosis, lead to respectively the migration, the proliferation and the elimination of tips. Thus, as shown in Eq. (1a), a reaction-diffusion equation was applied to describe the variation of the tip density. The diffusion term denotes the migration of tips with a diffusion coefficient $D$. The two reaction terms, $S$ and $P$, represent the proliferation and the elimination of tips. As the hyphal extension is generated only by tip elongation, the amount of hyphae created in a unit volume over a short time 
interval is linearly related to the actual quantity of tips by two factors, the averaged tip elongation rate $R$ and the proportion of active tips $P_{\text {active }}$ (Eq. (1b)).

$$
\begin{gathered}
\frac{\partial C}{\partial t}=\nabla(D \nabla C)+S(B, C)-P(B, C), \\
\frac{\partial B}{\partial t}=R P_{\text {active }} C .
\end{gathered}
$$

As mentioned above, the term $S$ denotes the production of tips by lateral and apical branching. According to the branching mechanisms in our discrete model, the number of lateral branches emerged per unit of time is the product of lateral branching probability $P_{b r}^{l a t}$ by the total quantity of tips in the mycelium. The latter is obtained as the integral of the tip density over all the entire volume (i.e., $\int C d \mathbf{x}$ ). The lateral branches should be uniformly distributed in the mycelium. Thus, the amount of branches distributed into each unit volume depends on the proportion of hyphae containing in this volume over the whole mycelium (i.e., $B / \int B d \mathbf{x}$ ). In addition, the lateral branching is inhibited in two special cases related to the number of branches and tips per unit hyphal length. As shown in Eq. (2), we applied an exponential function to account for this inhibition, where $\beta$ is defined as the inhibition coefficient and $B r(\mathbf{x}, t)$ denotes the number of branches per unit volume at position $\mathrm{x}$ and time $t$. Since the branches do not move, $B r$ is the accumulation of the term $S$ over time, calculated by Eq. (3).

$$
\begin{gathered}
Y(\mathbf{x}, t)= \begin{cases}e^{-\beta(C(\mathbf{x}, t)+B r(\mathbf{x}, t)) / B(\mathbf{x}, t)} & B(\mathbf{x}, t)>0, \\
0 & B(\mathbf{x}, t)=0 .\end{cases} \\
\frac{\partial B r}{\partial t}=S .
\end{gathered}
$$

For apical branching, each active tip possesses a probability $P_{b r}^{a p i}$ to form a new tip. The increase of tips due to the apical branching is hence given by $P_{b r}^{a p i} P_{a c t i v e} C$. Considering that the apical branching occurs when the hypha attains a certain length, we modified $C(\mathbf{x}, t)$ with a timedelayed term $C(\mathbf{x}, t-\tau)$ where $\tau$ is the necessary time for a tip to reach that given length with the average elongation rate. Together, the proliferation term $S$ is modeled as:

$$
S(B, C)=P_{b r}^{l a t} \int C d \mathbf{x} \frac{B}{\int B d \mathbf{x}} Y+P_{b r}^{a p i} P_{a c t i v e} C(\mathbf{x}, t-\tau) .
$$

The disappearance of tips is caused by anastomosis. Such a fusion occurs when a hyphal tip contacts another hypha, which implies that the disappearance rate depends on the contact 
probability between tips and hyphae. Since tips elongate at a low rate $\left(\sim 10^{-9} \mathrm{~m} \cdot \mathrm{s}^{-1}\right)$, they move a very short distance during a unit time interval. We focused on one tip and we took the volume formed by a unit surface and the short distance the tip passes through during a unit time interval. All the hyphae contained in this volume were projected on the bottom surface. The possibility of this tip contacting another hypha is equal to the proportion of the projected area, which is related to the hyphal length. In all, the elimination rate of tips is proportional to the tip density $C$, the tip elongation rate $R$, and the hyphal density $B$ and can be expressed as follows:

$$
P(B, C)=\gamma R B C
$$

The coefficient $\gamma$ is named as the anastomosis coefficient for correlating the projected area with the hyphal density.

To summarize, the reaction-diffusion system takes the following form

$$
\begin{aligned}
\text { Tip density } & \frac{\partial C}{\partial t}=\nabla(D \nabla C)+S-P, \\
\text { Hyphal density } & \frac{\partial B}{\partial t}=R P_{\text {active }} C, \\
\text { Branch density } & \frac{\partial B r}{\partial t}=S .
\end{aligned}
$$

where

$$
\begin{gathered}
S=\left\{\begin{array}{cc}
P_{b r}^{l a t} \int C d \mathbf{x} \frac{B}{\int B d \mathbf{x}} e^{-\beta(C+B r) / B}+P_{b r}^{a p i} P_{\text {active }} C(\mathbf{x}, t-\tau) & B(\mathbf{x}, t)>0, \\
P_{b r}^{a p i} P_{a c t i v e} C(\mathbf{x}, t-\tau) & B(\mathbf{x}, t)=0,
\end{array}\right. \\
P=\gamma R B C .
\end{gathered}
$$

\subsection{Numerical solution}

The model was solved in a one-dimensional (1D) axisymmetric configuration to be compared with the radial profiles obtained with the discrete model. The 1D axisymmetric formulation reads as follows:

$$
\begin{aligned}
& \frac{\partial C(r, t)}{\partial t}=\frac{1}{r} \frac{\partial}{\partial r}\left(D r \frac{\partial C(r, t)}{\partial r}\right)+S-P \\
& \frac{\partial B(r, t)}{\partial t}=R P_{\text {active }} C \\
& \frac{\partial B r(r, t)}{\partial t}=S .
\end{aligned}
$$


The term $C(\mathbf{x}, t-\tau)$ in Eq. (9a) is approximately transformed to an term able to be solved:

$$
C(t-\tau) \approx C(t)-\tau \frac{\partial C}{\partial t}(t)
$$

The initial condition for this model was defined as the averaged density profiles of tips, hyphae and branches obtained with the discrete model at $t=2.25 \times 10^{5} \mathrm{~s}$.

The reaction-diffusion system was solved in cylindrical coordinates with a computational domain large enough to assume no-flux boundary conditions. A control-volume finite-element spatial discretization turned the PDEs system (Eq. (10)) into an initial value problem of the form

$$
\frac{d u}{d t}=g(u), u(0)=u_{0}
$$

where $u=(C, B, B r) \in \mathbb{R}^{3 N}, g: \mathbb{R}^{3 N} \subset D \rightarrow \mathbb{R}^{3 N}$ is a nonlinear function of $u$, and $N$ is the size of the $1 \mathrm{D}$ mesh. The calculation domain was meshed uniformly with a spacing of $1.5 \times 10^{-4} \mathrm{~m}$ which is equal to the spatial resolution of the profiles obtained in the discrete model.

We performed the time integration process to get a linearized version of Eq. (12),

$$
\frac{d u}{d t}=g\left(u_{n}\right)+J_{g}\left(u_{n}\right)\left(u-u_{n}\right), t>t_{n}
$$

whose exact solution is

$$
u(t)=u_{n}+\left(t-t_{n}\right) \varphi\left(\left(t-t_{n}\right) J_{g}\left(u_{n}\right)\right) g\left(u_{n}\right), t>t_{n}
$$

where $J_{g} \in \mathbb{R}^{3 N \times 3 N}$ is the Jacobian matrix of $g$ and function $\varphi$ is defined as $\varphi(z)=\frac{e^{z}-1}{z}$. of Eq. (12)

$$
u_{n+1}=u_{n}+d t_{n} \varphi\left(d t_{n} J_{g}\left(u_{n}\right)\right) g\left(u_{n}\right)
$$

with time step $d t_{n}=t_{n+1}-t_{n}$.

The exponential integrators method was first presented in 1960 by Certaine [26] and has been well-known since late of 1990s by works of M. Hochbruck and others [27, 28]. Thanks to the approximation of the Krylov subspace to the matrix-function vector product $\varphi\left(d t_{n} J_{g}\left(u_{n}\right)\right) g\left(u_{n}\right)$, the exponential integrators method became very useful for large system of stiff equations [29, 30, 31]. 
In this article, our work was inspired from the work of E. Carr, I.Turner and P. Perré [30] using the "variable-stepsize exponential Euler method" to solve the system of non-linear transport equations with great success.

The Krylov subspace methods compute a small $m \times m$ matrix $H_{m}$ (the projection matrix) instead of the full Jacobian matrix $J_{g}$. The approximation of the matrix-vector product is as follows:

$$
\varphi\left(d t_{n} J_{g}\left(u_{n}\right)\right) g\left(u_{n}\right) \approx \beta_{0} V_{m} \varphi\left(d t_{n} H_{m}\right) e_{1} .
$$

Thanks to this, we can have a large time step $d t_{n}$ without preconditioning techniques, which is very efficient in terms of computational time and RAM memory.

\subsection{Parameter determination}

The continuous model was calibrated for P. Placenta via the validated discrete model mentioned in Part 2.1. All parameters are summarized in table 1 using the international unit standard. Most of these parameters were defined directly from the discrete model [25]. For example, since the tip elongation rates follow a corrected gamma distribution, its mean was calculated as the averaged tip elongation rate $R$. With this averaged rate, we obtained $\tau \approx 1048 \mathrm{~s}$, which is the average time for a tip to extend by $5 \mu \mathrm{m}$. The proportion of active tips, the lateral and the apical branching probability are simply those of the discrete model.

The determination of the three remaining, macroscopic parameters, the diffusion coefficient $D$, the inhibition coefficient $\beta$ and the anastomosis coefficient $\gamma$, were determined by inverse analysis. The objective function used for optimization compares, in the sense of mean squared difference, the radial profiles obtained with the discrete and continuous models. Since the tip and the hyphal density are tightly related and our final objective is to fit the latter one, we defined the relative error $E(B)$ of the hyphal density profiles:

$$
E(B)=\sqrt{\frac{\sum_{t \in\left\{t_{1}, \ldots, t_{9}\right\}} \sum_{k=1}^{N}\left(\rho_{\text {hypha }}[r(k), t]-B[r(k), t]\right)^{2}}{\sum_{t \in\left\{t_{1}, \ldots, t_{9}\right\}} \sum_{k=1}^{N} \rho_{h y p h a}^{2}[r(k), t]}},
$$

where the total number of radius increments is denoted by $N$ (here $N=299$ ) and $r(k)$ represents the radius of the $k^{t h}$ ring. This function was minimized using the Nelder-Mead method. 
Table 1. Description of variables and parameter values in the continuous model.

\begin{tabular}{cccl}
\hline Symbol & Description & Unit & Value \\
\hline$C$ & Tip density & $m^{-3}$ & -- \\
$B$ & Hyphal density & $m \cdot m^{-3}$ & -- \\
$B r$ & Branch density & $m^{-3}$ & -- \\
$t$ & Time & $s$ & -- \\
$R^{*}$ & Tip elongation rate & $m \cdot s^{-1}$ & $4,77 \times 10^{-9}$ \\
$P_{a c t i v e}^{*}$ & Proportion of active tips & -- & 0,7 \\
$P_{b r}^{l a t *}$ & Lateral branching probability & $s^{-1}$ & $1.11 \times 10^{-5}$ \\
$P_{b r}^{a p i *}$ & Apical branching probability & $s^{-1}$ & $3.22 \times 10^{-6}$ \\
$\tau^{*}$ & Lag time for apical branching & $s$ & 1048 \\
$D$ & Diffusion coefficient & $m^{2} \cdot s^{-1}$ & $1.13 \times 10^{-12}$ \\
$\beta$ & Inhibition coefficient & -- & $7.78 \times 10^{-5}$ \\
$\gamma$ & Anastomosis coefficient & $m$ & $5.40 \times 10^{-2}$ \\
\hline
\end{tabular}

* Parameter values come from [25].

\section{Results} discrete model (Fig. 2). The profiles confirm that, after a long-term growth, the tip density is almost uniformly distributed, even though it decreases slightly towards the center of the colony. The higher distribution of tips at the edge is a strategy that favors the exploitation of new space. The uniform distribution implies the existence of an equilibrium between the emergence of branches to simulate the tip movement in a continuum approach. One may note also that the tip density in the center increases first and then decreases at longer times. Indeed, when the mycelial network becomes dense, the anastomosis and the inhibition occur more frequently, leading to more elimina- 
tion of tips. From Fig. 2 (b), we observed that the hyphal density profiles continuously increased in time keeping a negative gradient towards the edge. Note however that the hyphal density increases more and more slowly in the center due to the reduction of tip density, revealing the existence of an upper limit to the biomass even for the free growth of mycelia on a two-dimensional substrate.

(a)

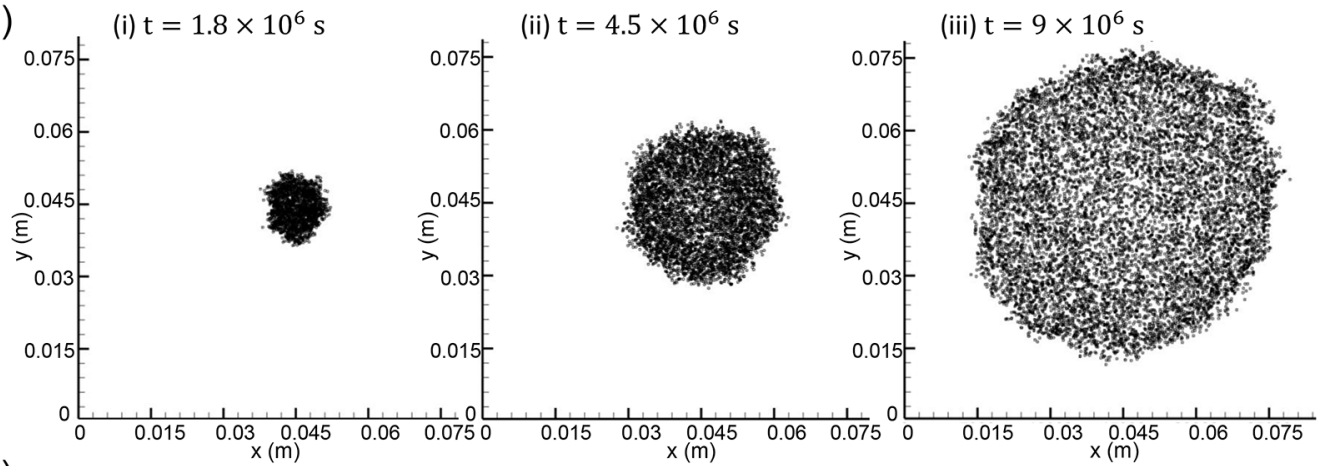

(b)

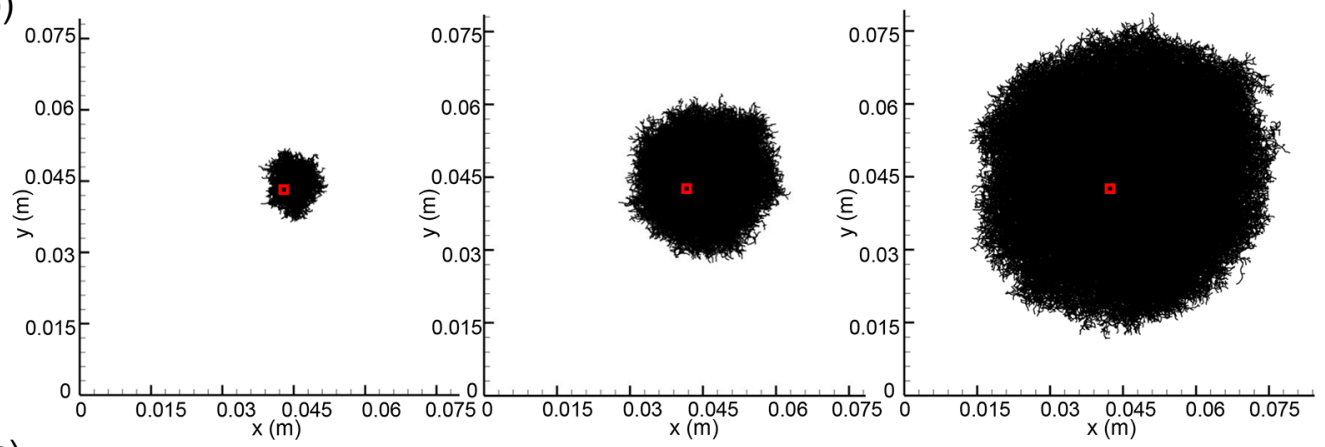

(c)

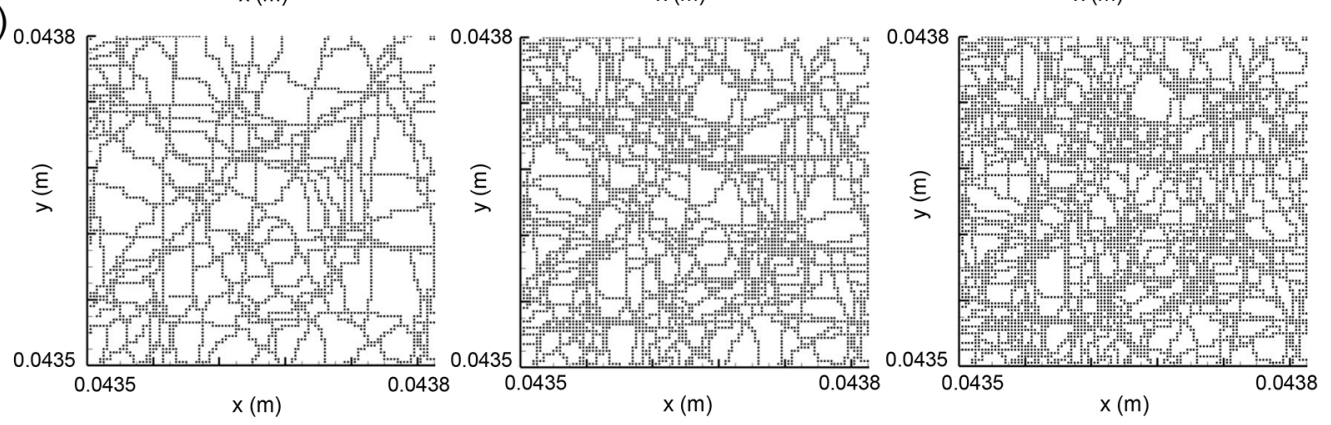

Fig. 1. Simulation of the discrete model: temporal evolution of (a) the distribution of tips, (b) the mycelial network and (c) the zoom of the red squared part in (b).

\subsection{Validation of the PDE formulation}

The profiles averaged over 20 realizations of the stochastic model (Fig. 2) were used to optimize the three macroscopic parameters of the continuous model. The optimized solution of Eq. (10) has a very small relative error $\left(E(B)=2.70 \times 10^{-2}\right)$, which proves the ability of the PDE formulation to represent the discrete mechanisms. The profiles, plotted at different times in Fig. 3, give another piece of information: even though the objective function considers only the hyphal density, both 
(a)

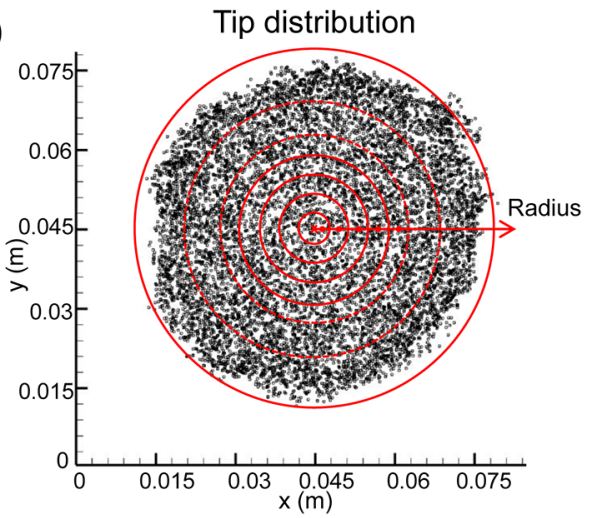

(b)

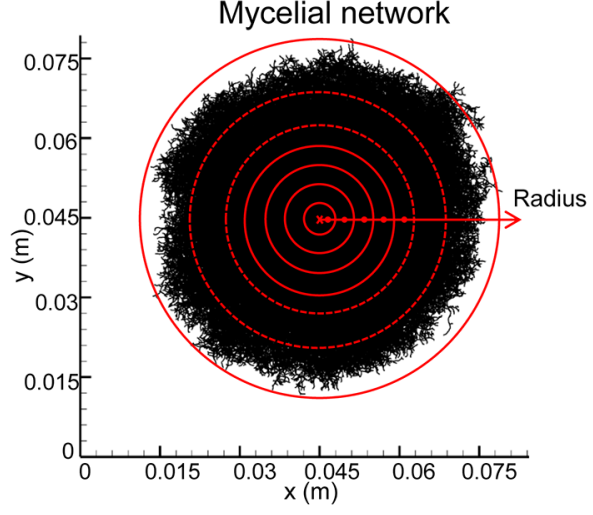

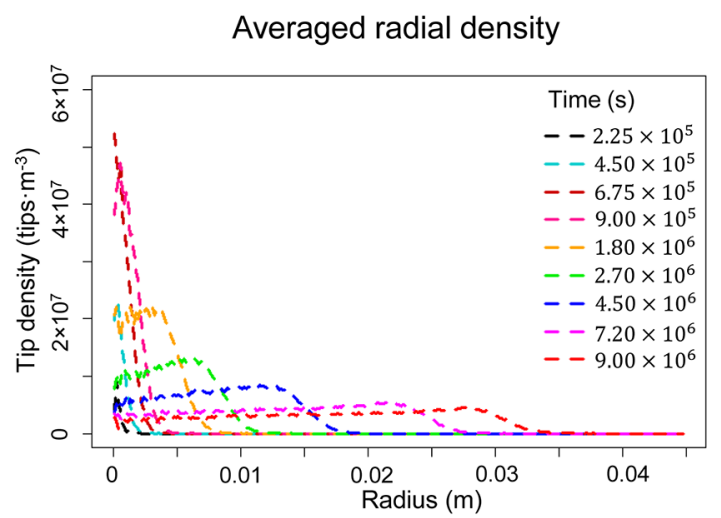

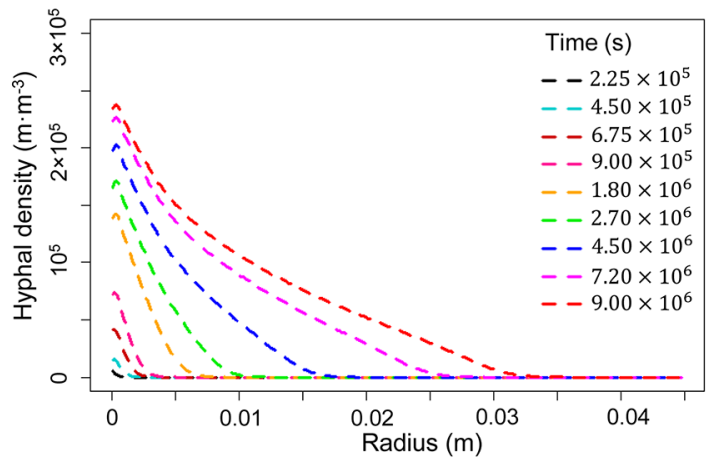

Fig. 2. Radial densities of tips and hyphae obtained by averaging respectively the tip distributions and the mycelial networks over radius increments at nine times. Profiles on the right are the average of 20 realizations of the stochastic discrete model. 
the tip and the hyphal profiles of the PDE solution are in excellent agreement with the discrete model. This confirms that the two variables, tip density and hyphal density, are tightly related to each other. Moreover, the wave-shape of the tip profiles was well captured by the diffusion process, even though it resembles more a front-like progression than a diffusion phenomenon.

235
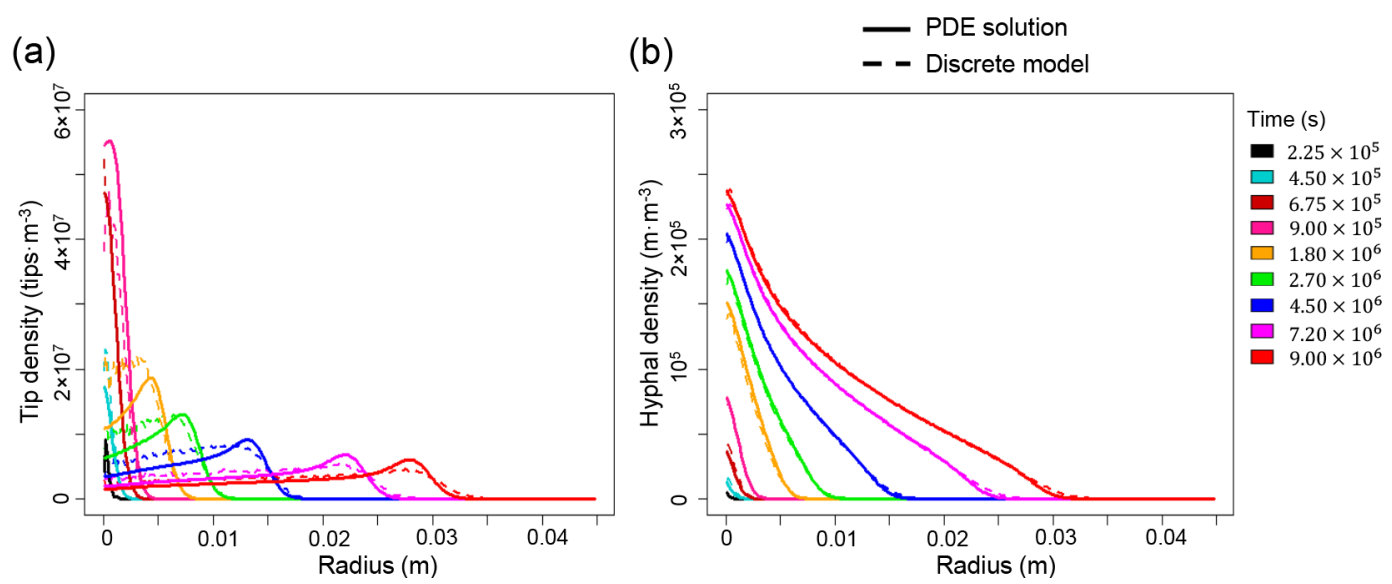

Fig. 3. Comparison of the numerical solutions (solid line) after optimization with the averaged radial profiles (dashed line) of the discrete model at eight times. The black solid lines depict the initial condition for the continuous model, which are the averaged profiles obtained with the discrete model at $t=2.25 \times 10^{5} \mathrm{~s}$.

Compared to the advection process, which requires the propagation direction to be known a priori, the diffusion process greatly facilitates multi-dimensional modeling. Indeed, as the propagation results are from a diffusion process, the propagation direction is defined locally from the actual gradient of the tip concentration. The other discrete mechanisms are also well described by the continuous terms, even the implicit ones. This is the case in particular for the anastomosis rate at the macroscale, assumed to be linearly related to the tip density, the tip elongation rate and the hyphal density, and for the branching inhibition expressed as an exponential function depending on the branching and tip density per unit length of hyphae. The value of the three parameters (i.e., $D, \beta$ and $\gamma$ ) determined by inverse procedure are listed in Table 1.

\subsection{Ability of the PDE model to simulate different growth conditions}

In the previous section, we proved that the continuous model perfectly reproduces, at the macroscopic scale, the growth of $P$. Placenta for the reference parameter set of the discrete model $([24,25])$. However, the change of the growth conditions, such as temperature, humidity or nutrient concentration, influences the behavior of hyphal growth, particularly branching and elongation rate $[32,33,34,35]$. It is therefore very important to check whether the continuous model is able to predict the growth behaviour for different parameters of the discrete model. To that purpose, we 
altered the three key parameters of hyphal growth in the discrete model, (i.e., the apical branching probability $P_{b r}^{a p i}$, the lateral branching probability $P_{b r}^{l a t}$ and the tip extension rate $R$ ). The values of the three parameters listed in Table 1 were considered as reference. Then, for each parameter, we performed simulations with $0.5,1.5$ and 2 times the reference value. These factors were arbitrarily chosen to represent a quite significant change of growth conditions. This means that nine series of simulations (20 realizations per case study) have been carried out by changing one parameter of the discrete model at a time. The corresponding profiles were obtained in the same way as described in Part 2.1.

Obviously, these changes were reported when these discrete parameters are involved explicitly in the continuous formulation. The three macroscopic parameters identified in the previous section were used as initial guess. Then, for each series of profiles, we optimized four times the objective function $E(B)$ (Eq. (17)) by using different set of free parameters $(D, \gamma, \beta),(D, \gamma),(D, \beta)$ and $D$. The relative errors of the hyphal density between the discrete and continuous solutions are presented in Fig. 4 before and after optimization. According to this bar chart, a good continuous-discrete match requires the value of $D$ to be modified when the discrete parameter $P_{b r}^{a p i}$ changed, while both $D$ and $\gamma$ should be adjusted when parameters $P_{b r}^{l a t}$ and $R$ changed. Accordingly, parameter $\beta$ can be kept unchanged in any case. The relative errors of the optimal solutions are all around $4.00 \times 10^{-2}$, which is low enough to ensure the capacity of the continuous model to replicate the mycelial growth under different conditions.

To better understand the impact of the three discrete parameters on the mycelial growth, we compared the profiles of the tip and the hyphal density obtained with different values of $P_{b r}^{a p i}, P_{b r}^{l a t}$ and $R$ (respectively Fig. 5, 6 and 7) after optimization. In accordance with the results of Fig. 4, $D$ was the unique adjusted parameter to match the changes of $P_{b r}^{a p i}$ while two parameters, $D$ and $\gamma$, were adjusted when changing the value of $P_{b r}^{l a t}$ or $R$. The numerical solutions agree well with the discrete results for all of the nine cases. With the increase of the apical and lateral branching probability, the local tip and hyphal density increase more rapidly since the proliferation rate of tips is higher. As a subtler effect, we may notice that the extension rate of the colony also augments with the increase of local tips. This result is from a statistical effect: with the increase of tips at the edge of the mycelium, the chance to have tips whose extension direction is close to the radial direction also increases, resulting in a more efficient spatial extension of the network. With the increase of the tip elongation rate, the extension rate of the colony rises but the local hyphal density reduces significantly. Since the tip proliferation rate does not change, the amount of tips produce by branching is similar, but, as the colony occupies a larger space, less tips will be distributed per unit volume. 


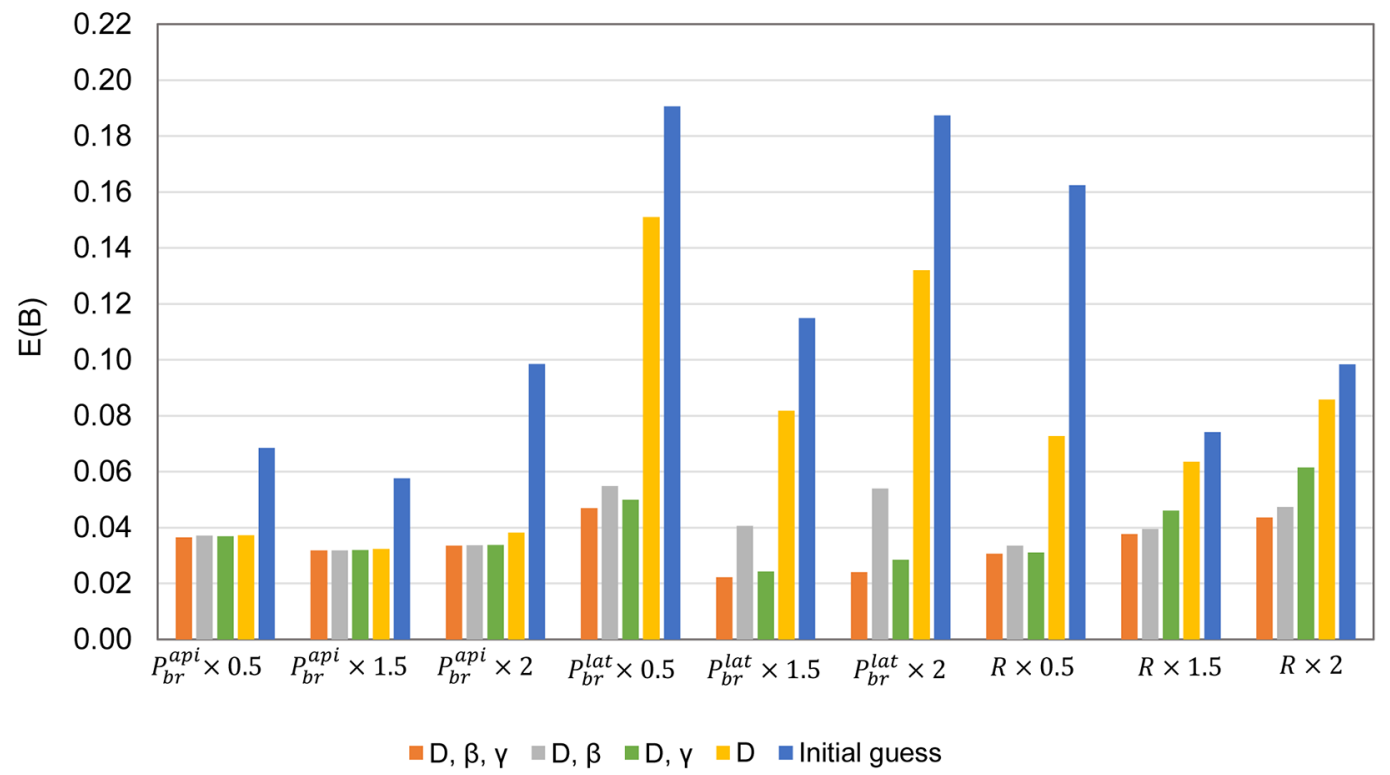

Fig. 4. Relative errors of the hyphal density $(E(B))$ between the discrete profiles and the numerical solutions in nine cases (in each case we varied one growth parameter in the discrete model). The five colors represent the numerical solutions before the optimization (blue) and after the optimization executed in specifying $D, \beta$ and $\gamma$ (orange), $D$ and $\beta$ (grey), $D$ and $\gamma$ (green), and $D$ (yellow).

(a)
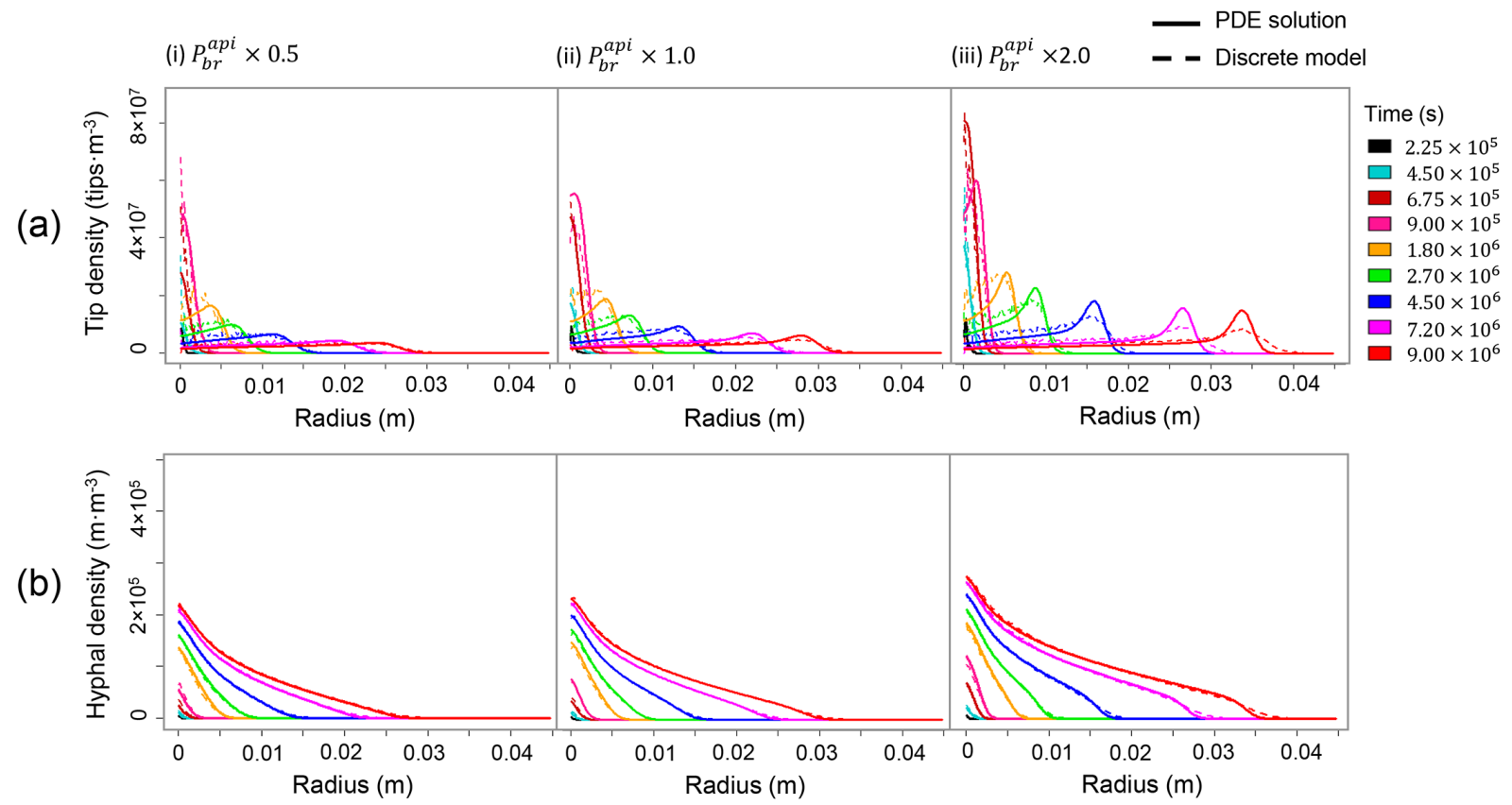

Fig. 5. Comparison of the numerical solutions after the optimization in identifying $D$ and the radial profiles in the three cases of the variation of the apical branching probability $P_{b r}^{a p i}$ in the discrete model.

To summarize, even though the impact of $P_{b r}^{l a t}$ and $R$ is greater than that of $P_{b r}^{a p i}$, the three parameters influence the morphology of the mycelial network by intricate effects. This necessarily 


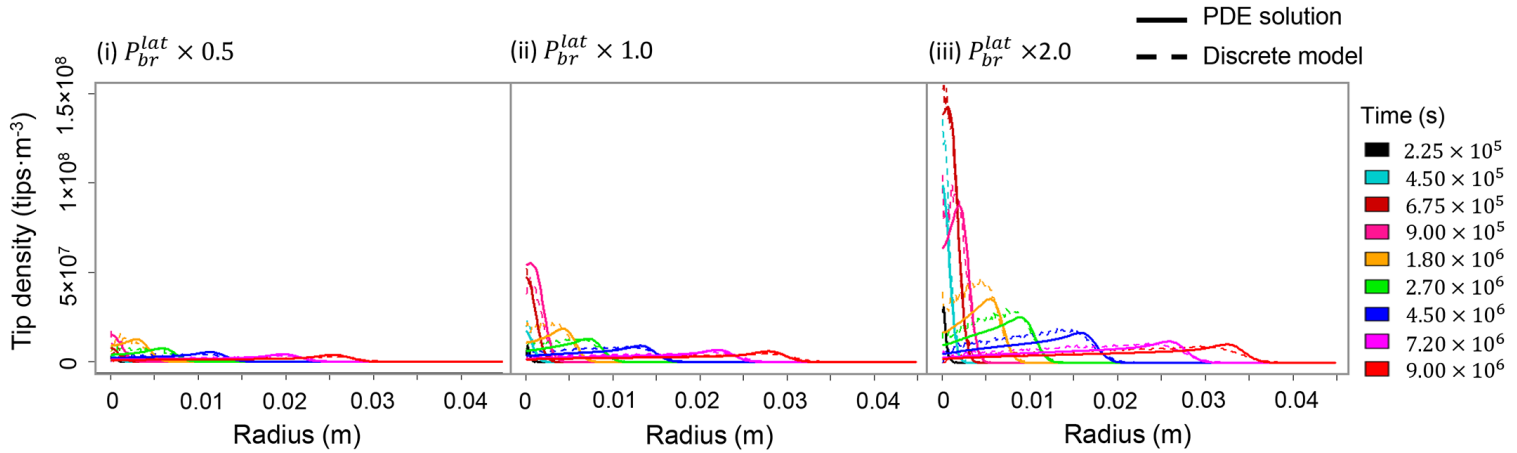

(b)

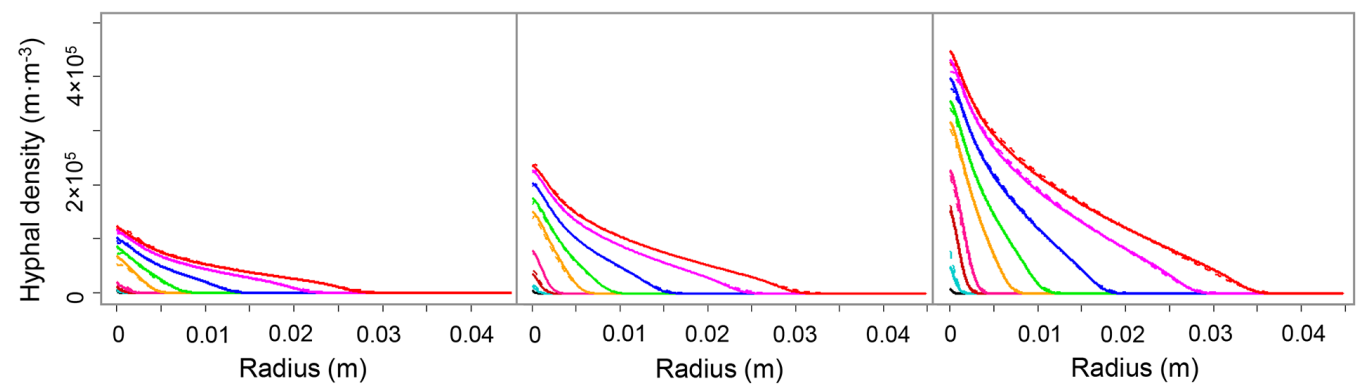

Fig. 6. Comparison of the numerical solutions after the optimization in identifying $(D, \gamma)$ and the radial profiles in the three cases of the variation of the lateral branching probability $P_{b r}^{l a t}$ in the discrete model.

(a)

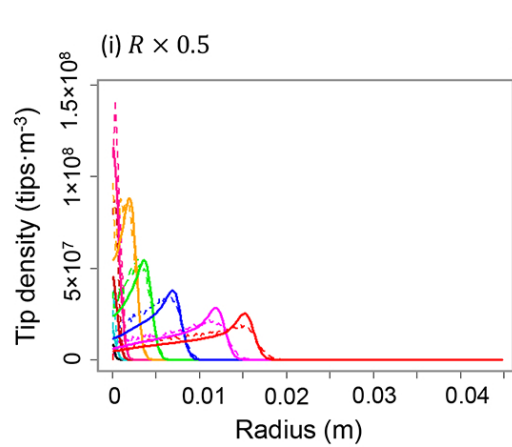

(ii) $R \times 1.0$

(iii) $R \times 2.0$

- PDE solution
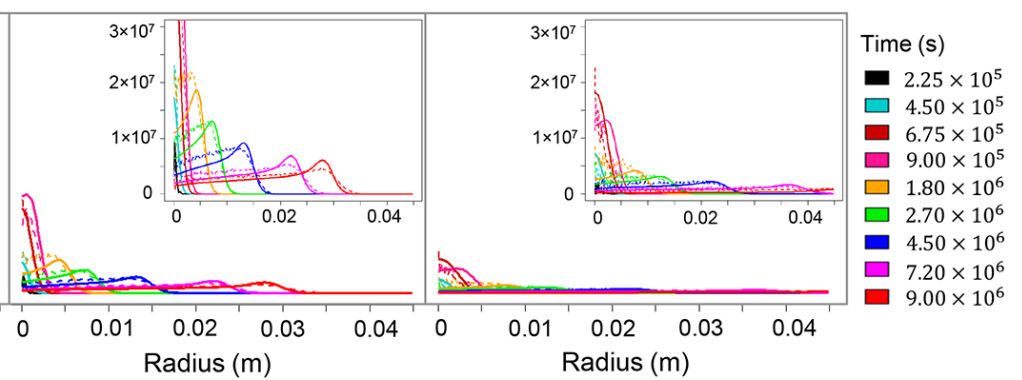

(b)
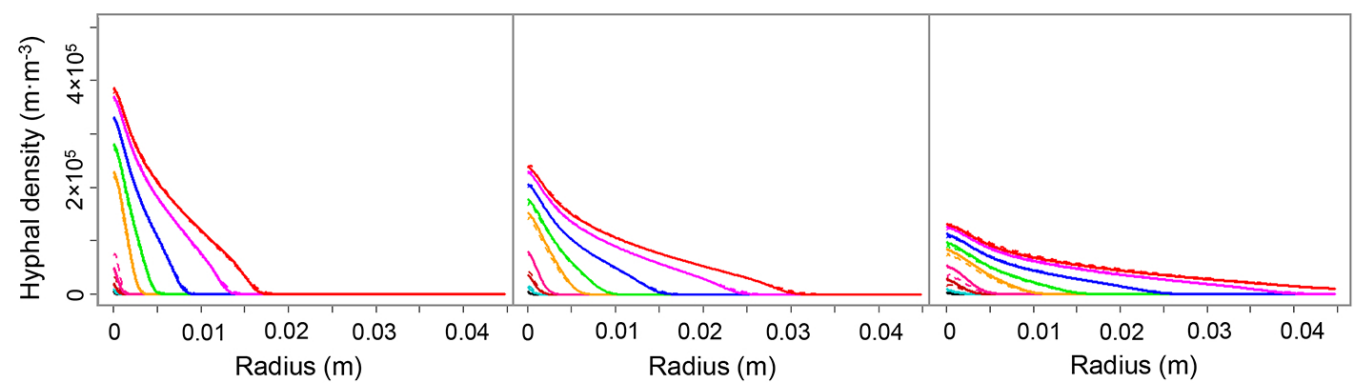

Fig. 7. Comparison of the numerical solutions after the optimization in identifying $(D, \gamma)$ and the radial profiles in the three cases of the variation of tip elongation rate $R$ in the discrete model. 
modifies the colony size and the spatio-temporal distribution of biomass, which explains why the global parameters of the PDE model, the diffusion coefficient $D$ and/or the anastomosis coefficient $\gamma$, need to be adjusted. The effect of the three growth parameters on the macroscopic coefficient $D$ or $\gamma$ are depicted in Fig. 8. The fitted expressions proposed in this figure have all a coefficient of determination $r^{2}$ close to the unit.
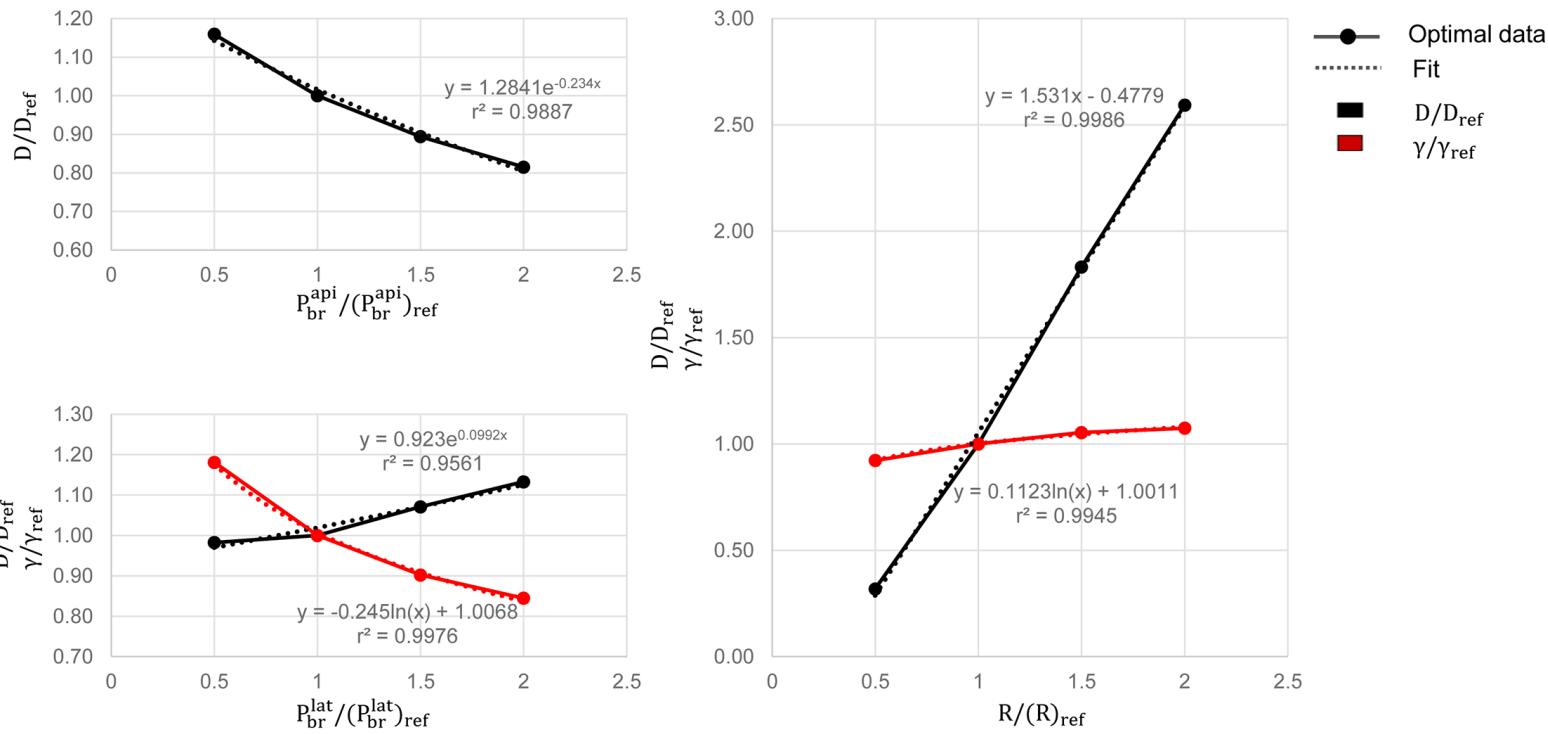

Fig. 8. Relationship between $D$ or $\gamma$ and the three growth parameters. $X_{\text {ref }}$ represents the reference value of each parameter listed in Table 1. Dots are the optimal values of $D$ or $\gamma$ obtained for different growth parameters. Dash lines are the smoothing functions.

Consistently, the tip elongation rate $R$ has a direct impact on $D$. The effect of $R$ is almost linear with a slope equal to 1.5 . The fact that $D$ varies more rapidly than $R$, can be explained by the indirect effect of tip elongation rate on tip density.

The effect of branching probabilities on $D$ reveals more subtle mechanisms. Let's focus first on the negative correlation between apical branching probability $P_{b r}^{a p i}$ and $D$. We have to keep in mind that a higher value of $P_{b r}^{a p i}$ slightly increases the colony expansion rate, because the probability to have active tips right along the macroscopic radial direction increases with the tip density. Besides, the tip density at the colony edge is higher, giving rise to a larger tip gradient. Consequently, the diffusivity $D$ must be smaller to obtain the right expansion rate.

This coupled effect of tip gradient and diffusivity to build up the macroscopic extension rate is also likely to explain the positive correlation between lateral branching probability $P_{b r}^{l a t}$ and $D$. Due to apical inhibition, a higher lateral branching probability increases the tip density everywhere 
except right at the colony edge. This non-uniform tip source term tends to decrease the tip gradient at the colony edge and should be compensated by a slightly larger diffusivity $D$.

Regarding the effect of growth condition on $\gamma$, equation $9 \mathrm{~b}$ reminds us that this factor corrects tition of biomass $B$ in a volume, the projected area along tip extension is not simply proportional to $B$. In a certain volume, the proportion of overlapping hyphae increases with density. When $P_{b r}^{l a t}$ increases or $R$ decreases, the hyphal density increases and $\gamma$ should be reduced to obtain the correct anastomosis term.

\section{Discussion}

As summary of this work, it is worth to come back to our global upscaling strategy of mycelial growth modeling. We first proposed a discrete model which mimics the mycelium development at the microscopic scale [25]. This model accounts for the fundamental mechanisms of network development (tip extension, lateral and apical branching, anastomosis). It has been calibrated and validated from experimental data by a rigorous procedure. For that purpose, the network development has been observed during 17 days by confocal microscopy, and the image were subsequently processed to extract relevant information [24]. In the discrete model, some parameters such as the lateral and apical branching probabilities, were determined by an inverse analysis. Without the discrete model, these parameters could not have been accurately determined from experimental data. Moreover, via the validation process, some underlying mechanisms have been discovered, such as the inhibition of branching which is almost impossible to observe directly from the mycelial morphology.

Acting as a bridge between the microscopic behaviors of hyphal growth and the mycelial develduring $\sim 104$ days. This is quite computationally intensive as one realization requires about 50 Go of RAM memory and a computational time of ca. 8 hours on a single core of a HPC cluster with the processor Intel Xeon CPU E5-2670 v3 @ $2.30 \mathrm{GHz}$. By this way, the spatial dimension was extended from $0.9 \mathrm{~cm} \times 0.9 \mathrm{~cm}$ (experiment) to $9 \mathrm{~cm} \times 9 \mathrm{~cm}$ (simulation). Therefore, the discrete model already allowed the spatio-temporal scale to be efficiently extended.

20 realizations of the discrete model, ca. 160 hours of CPU time, were averaged to obtain tip/hyphal density profiles. This piece of information served as input data for the continuous model proposed in the present work. Based on the hyphal growth mechanisms at the discrete 
scale, a new reaction-diffusion system, involving three spatio-temporal variables (tip, mycelium and branch densities), has been derived. While most discrete parameters are involved explicitly in the continuous formulation, three macroscopic parameters had to be defined by inverse analysis. Once done, the discrete profiles are perfectly reproduced, in space and in time, by the PDE model. Thanks to the exponential Euler method with Krylov subspace approximation, the computational time to solve the continuous model was again greatly compressed to less than $4 s$ on a single core of the same cluster. In brief, by following the upscaling strategy, the continuous model realizes the prediction of the mycelial growth during 104 days in less than $4 s$. In addition, since the memory required by the continuous model is much less than the discrete model, it is possible to further enlarge, by several orders of magnitude, the spatial and time scales.

Our continuous model is based on a reaction-diffusion formulation. Unlike advection models, it is not necessary for the diffusion process to know the development direction at each point a priori. This feature opens the door to multi-dimensional modeling and growth simulation in porous media. For the reaction part, we rigorously followed the mechanisms of the discrete model, which implies that all parameters have a physical meaning. In addition, thanks to the balance between diffusion and production (a global effect of branching), an almost constant gradient establishes at the edge of the network. This can be seen at the edge of the colony in Fig. 3(a): a tip density peak develops towards the free space. Therefore, an almost constant propagation rate arises from this diffusion-reaction mechanism. Consequently, a constant diffusivity is likely to simulate a given average tip elongation rate. The reaction-diffusion process is applied to the tip density, while the hyphal density is the accumulation of the tip density over time. Similarly, the branch density at any location is obtained as the time integration of the source terms of branches at that location. The 3-variable PDE model involves coupling between variables. For example, the tip density is connected to the hyphal density as the latter influences the lateral branching distribution and the anastomosis. Even so, the relation between the two main densities (tips and biomass) is nicely reproduced by the continuous model.

Apart from the reference simulation, additional series of profiles were produced by the discrete model with various parameter values, which are artificially set just to give us insight into the link between the possible microscopic mechanisms and the correponding macroscopic behaviours. These configurations have been reproduced by the continuous model. Whether the branching rate or the elongation rate has great impact on the morphology of the mycelium by changing the colony extension rate and the local biomass distributions. They can also alter the maximal value of the tip density, revealing that they change the balance between branching and anastomosis. In addition, 

mycelial morphology and the biomass distribution. On the contrary, the variation of the local mechanism and the mycelial network can also be estimated via the experimental observation of the biomass distribution together with an inverse procedure. In this sense, the numerical efficiency of the continuous model is of particular interest.

\section{Conclusion}

This work proposes a new three-variable reaction-diffusion PDE model capable of simulating and predicting the mycelial growth under different environmental conditions. This model was calibrated using variable profiles predicted by the average over several realizations of a stochastic formulation rigorously follows the discrete mechanisms of mycelial development, which implies that all parameters have a physical meaning. Thanks to the discrete model, the ability of this new model to account for various growth conditions was successfully tested. Moreover, the correlation between the discrete and continuous model has been constructed, which provides us a tool to realize the bidirectional transfer, from the local biological mechanisms to the global biomass distribution and vice-versa.

As the mycelium network propagation is formulated as a diffusion process, this model has the potential to be easily extended in multi-dimensions and to heterogeneous media such as a Low Density Fiberboard. In the future, additional features such as translocation and substrate-degradation ogy of bio-based materials as obtained by nano-tomography. Once combined with decomposition mechanisms, this model can also be applied to solid-state fermentation. 


\section{Acknowledgments}

This work has benefited from the financial support of the LabeX LaSIPS (ANR-10-LABX-0040LaSIPS) managed by the French National Research Agency under the "Investissements d'avenir" program (nANR-11-IDEX-0003-02). The calculus presented in this work were performed on the Fusion HPC Center, CentraleSupélec, Université Paris-Saclay and on the ROMEO HPC Center, Université de Reims Champagne-Ardenne. Financial supports from Grand Reims, the Marne de-

\section{References}

[1] O. Schmidt, Wood and Tree Fungi, Springer Verlag Berlin Heidelberg, 2005. doi:10.1007/ 3-540-32139-X.

[2] B. Jin, T. Schultz, D. Nicholas, Structural characterization of brown-rotted lignin, Holzforschung 44 (2) (1990) 133-138.

[3] K. Eriksson, R. Blanchette, P. Ander, Microbial and Enzymatic Degradation of Wood and Wood Components., Springer Berlin Heidelberg, 1990.

[4] B. Goodell, J. Jellison, J. Liu, G. Daniel, A. Paszczynski, F. Fekete, S. Krishnamurthy, L. Jun, $\mathrm{G}$. Xu, Low molecular weight chelators and phenolic compounds isolated from wood decay fungi and their role in the fungal biodegradation of wood, J. Biotechnol. 53 (2-3) (1997) 133-162. doi: 10.1016/S0168-1656(97)01681-7.

[5] D. Yelle, D. Wei, J. Ralph, K. Hammel, Multidimensional NMR analysis reveals truncated lignin structures in wood decayed by the brown rot basidiomycete Postia placenta., Environ Microbiol. 13 (4) (2011) 1091-1100. doi:10.1111/j.1462-2920.2010.02417.x.

[6] Y. Xie, J. Bjurman, L. Wadsö, Microcalorimetric characterization of the recovery of a brownrot fungus after exposures to high and low temperature, oxygen depletion, and drying, Holzforschung 51 (3) (1997) 201-206. doi:10.1515/hfsg.1997.51.3.201.

[7] J. Gonzalez, J. Morrell, Effects of environmental factors on decay rates of selected white- and brown-rot fungi, Wood Fiber Sci. 44 (4) (2012) 343-356.

[8] L. Wadsö, S. Johansson, A. Pilgård, G. Alfredsen, The activity of rot fungi (Postia placenta) during drying and rewetting cycles measured by isothermal calorimetry, Eng. Life Sci. 13 (6) (2013) 536-540. doi:10.1002/elsc.201200096. 
[9] G. Boswell, H. Jacobs, K. Ritz, G. Gadd, F. Davidson, The development of fungal networks in complex environments, Bull. Math. Biol. 69 (2007) 605-634. doi:10.1007/ s11538-005-9056-6.

[10] G. Boswell, Modelling mycelial networks in structured environments, Mycol. Res. 112 (2008) 1015-1025. doi:10.1016/j.mycres.2008.02.006.

[11] M. Fuhr, M. Schubert, F. Schwarze, H. Herrmann, Modelling the hyphal growth of the wooddecay fungus Physisporinus vitreus, Fungal Biol. 115 (9) (2011) 919-932. arXiv:1101.1747, doi:10.1016/j.funbio.2011.06.017.

URL http://dx.doi.org/10.1016/j.funbio.2011.06.017

[12] L. Edelstein, The propagation of fungal colonies: a model for tissue growth (1982).

[13] L. Edelstein, Y. Hadar, I. Chet, Y. Henis, L. Segel, A model for Fungal Colony Growth Applied to Sclerotium rolfsii, Journal of General Microbiology 129 (1983) 1873-1881. doi: 10.1099/00221287-129-6-1873.

[14] L. Edelstein, L. Segel, Growth and metabolism in mycelial fungi, J. Theor. Biol. 104 (2) (1983) 187-210. doi : 10.1016/0022-5193(83)90410-1.

[15] L. Edelstein-Keshet, B. Ermentrout, Models for branching networks in two dimensions, SIAM J. Appl. Math. 49 (4) (1989) 1136-1157. doi:10.1137/0149068. URL http://www . jstor.org/stable/10.2307/2102010

[16] F. Davidson, Modelling the qualitative response of fungal mycelia to heterogeneous environments, J. Theor. Biol. 195 (3) (1998) 281-92. doi:10.1006/jtbi.1998.0739.

URL http://www.ncbi.nlm.nih.gov/pubmed/9826484

[17] F. Davidson, A. Park, A mathematical model for fungal development in heterogeneous environments, Appl. Math. Lett. 11 (6) (1998) 51-56. doi:10.1016/S0893-9659(98)00102-5.

[18] F. Davidson, S. Olsson, Translocation induced outgrowth of fungi in nutrient-free environments., J. Theor. Biol. 205 (1) (2000) 73-84. doi:10.1006/jtbi.2000.2045.

URL http://www.ncbi.nlm.nih.gov/pubmed/10860701

[19] R. Falconer, J. Bown, N. White, J. Crawford, Biomass recycling and the origin of phenotype in fungal mycelia., Proc. Biol. Sci. 272 (July) (2005) 1727-1734. doi:10.1098/rspb.2005.3150.

[20] R. Falconer, J. Bown, N. White, J. Crawford, Modelling interactions in fungi., J. R. Soc. Interface 5 (23) (2008) 603-15. doi:10.1098/rsif.2007.1210.

URL http://www.pubmedcentral.nih.gov/articlerender.fcgi?artid=2621247\&tool= pmcentrez\&rendertype=abstract 
[21] N. Cunniffe, C. Gilligan, Scaling from mycelial growth to infection dynamics: a reaction diffusion approach, Fungal Ecol. 1 (4) (2008) 133-142. doi:10.1016/j.funeco.2008.10.007.

URL http://dx.doi.org/10.1016/j.funeco.2008.10.007

[22] G. Boswell, H. Jacobs, F. Davidson, G. Gadd, K. Ritz, Functional consequences of nutrient translocation in mycelial fungi., J. Theor. Biol. 217 (2002) 459-477. doi:10.1006/yjtbi.3048.

[23] G. Boswell, H. Jacobs, F. Davidson, G. Gadd, K. Ritz, Growth and function of fungal mycelia in heterogeneous environments, Bull. Math. Biol. 65 (2003) 447-477. doi:10.1016/ S0092-8240(03) 00003-X.

[24] H. Du, P. Lv, M. Ayouz, A. Besserer, P. Perré, Morphological Characterization and Quantification of the Mycelial Growth of the Brown-Rot Fungus Postia placenta for Modeling Purposes, PLoS One 11 (9) (2016) e0162469. doi:10.1007/s00253-015-6949-7.

URL https://hal-centralesupelec.archives-ouvertes.fr/hal-01256880

[25] H. Du, M. Ayouz, P. Lv, P. Perré, A lattice-based system for modeling fungal mycelial growth in complex environments, Physica A 511 (2018) 191-206. doi:10.1016/j.physa.2018.07.051.

[26] J. Certaine, The solution of ordinary differential equations with large time constants., Mathematical methods for digital computers (1960) 128-132doi:10.1111/j.1574-6941.2011. 01106.x.

[27] M. Hochbruck, C. Lubich, H. Selhofer, Exponential integrators for large systems of differential equations, SIAM J. Sci. Comput. 19 (5) (1998) 1552-1574. doi:10.1137/ S1064827595295337.

[28] M. Hochbruck, A. Ostermann, Exponential integrators, Acta Nemerica (2010) 209-286doi: $10.1017 / \mathrm{S} 0962492910000048$

[29] E. Carr, T. Moroney, I. Turner, Efficient simulation of unsaturated flow using exponential time integration., Appl. Math. Comput. 217 (2011) 6587-6596. doi:10.1016/j.amc.2011.01. 041.

[30] E. Carr, I. Turner, P. Perré, A variable-stepsize Jacobian-free exponential integrator for simulating transport in heterogeneous porous media : application to wood drying, Journal of Computational Physics 233 (2012) 66--82. doi:10.1016/j.jcp.2012.07.024.

[31] D. Michels, G. Sobottka, A. Weber, Exponential integrators for stiff elastodynamic problems., ACM Transactions on Graphics (TOG) 33 (2014) Article 7. doi:10.1145/2508462. 
[32] V. Meyer, M. Arentshorst, S. Flitter, B. Nitsche, M. Kwon, C. Reynaga-Peña, S. BartnickiGarcia, C. Van Den Hondel, A. Ram, Reconstruction of signaling networks regulating fungal morphogenesis by transcriptomics., Eukaryotic Cell 8 (2009) 1677-1691. doi:10.1128/EC. 00050-09.

[33] J. Rousk, E. Baath, Growth of saprotrophic fungi and bacteria in soil., FEMS Microbiol Ecol. 78(1) (2011) 17-30. doi:10.1128/EC.00050-09.

[34] H. Jacobs, G. Boswell, K. Ritz, F. Davidson, G. Gadd, Solubilization of calcium phosphate as a consequence of carbon translocation by Rhizoctonia solani., FEMS Microbiol Ecol. 40 (2002) 65-71. doi:10.1016/S0168-6496(02)00202-7.

[35] J. Dighton, Fungi in Ecosystem Processes, Marcel Dekker, Inc., New York, NY, USA, 2003. arXiv:arXiv: 1011.1669v3, doi:10.1201/9780203911440.

URL http://www.crcpress.com/product/isbn/9780824742447 


\section{Discrete model}

Tip distribution

Mycelial network
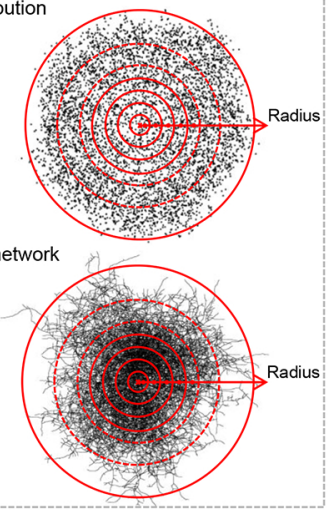

Continuous model

- Numerical solution

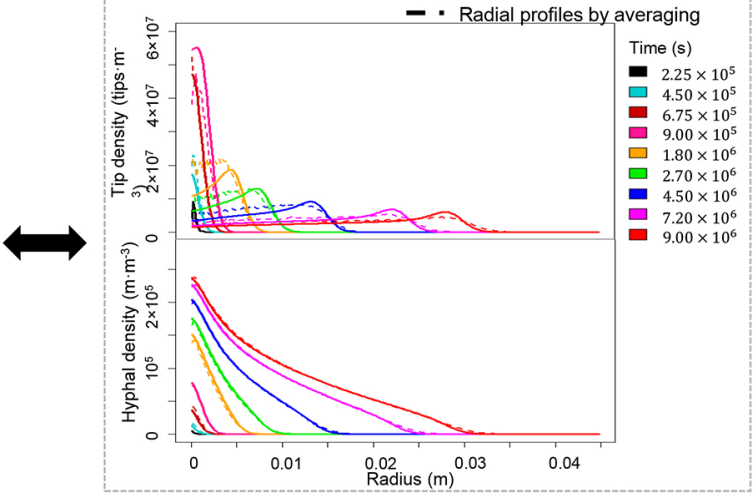

